\title{
Levantamento do Estado da Arte sobre Aprendizagem baseada em Problemas na Educação a Distância e Híbrida
}

\author{
Marcia de Oliveira Alves', Francisco Petrônio A. Medeiros' ${ }^{2}$, Lafayette B. Melo' \\ 'Programa de Pós-Graduação em Tecnologia da Informação - Instituto Federal da Paraíba \\ (IFPB) João Pessoa - PB - Brasil \\ \{marcia, petronio, lafayette\} a ifpb.edu.br
}

\begin{abstract}
This article presents a wide-ranging systematic literature review (RSL) on the adoption of the Problem Based Learning (PBL) methodology in Distance and Blended Education. The research analyzed primary studies from the years 2008 to 2019 to understand how the primary PBL methodology stages are applied, identify the most used Virtual Learning Environments (VLE) and tools, and the main impacts of PBL application in distance and blended education. A total of 633 studies were indexed using the search string, of which 77 were analyzed to answer the research questions based on the exclusion criteria. Some conclusions point out that for 75\% of the studies analyzed, the experiences presented are not associated with a VLE. Also, in the literature, there are few tool initiatives integrated or not to the AVAs that are specific to the planning, execution, or evaluation of $P B L$.
\end{abstract}

Resumo. Este artigo apresenta os resultados de uma ampla revisão sistemática da literatura (RSL) sobre a adoção da metodologia Aprendizagem baseada em Problemas (ABP) na Educação a Distância e Hibrida (EaDH). Foram analisados os estudos primários dos anos de 2008 a 2019 com o objetivo de refletir sobre como as principais etapas dessa metodologia estão sendo aplicadas na EaDH, quais os Ambientes Virtuais de Aprendizagem (AVA) e ferramentas mais utilizados, bem como os principais impactos da aplicação da metodologia ABP na EaDH. Um total de 633 estudos foram indexados a partir da string de busca, dos quais 77 foram analisados para responder às questões de pesquisa com base nos critérios de exclusão. Algumas conclusões foram que para 75\% dos estudos analisados, as pesquisas e experiências de ABP apresentadas não estão vinculados a um AVA e que há na literatura poucas iniciativas de ferramentas integradas ou não aos AVAs que sejam específicas ao planejamento, execução ou avaliação de $A B P$.

\section{Introdução}

Segundo Valente (2014), a democratização da Internet permitiu expandir o ensino na modalidade a distância e híbrida, requerendo urgência na atualização dos processos educativos. Este novo cenário propicia a aplicação de metodologias que, entre outros desafios, quebram o paradigma do professor como protagonista principal no processo educativo. Segundo de Souza Rabelo et al. (2018), a metodologia ativa Aprendizagem baseada em Problemas (ABP) tem sido cada vez mais utilizada no processo de ensinoaprendizagem, pois se baseia no princípio de utilização de problemas do mundo real com forte motivação prática e estímulo cognitivo para gerar soluções criativas. 
A metodologia ABP tende a privilegiar os conhecimentos prévios dos estudantes, incentivando um ambiente investigativo de aprendizagem através da construção de hipóteses e experimentações. Este trabalho se concentra na revisão sistemática da literatura acerca da adoção da metodologia ativa de Aprendizagem baseada em Problemas (ABP) na educação a distância e híbrida, com o objetivo refletir sobre como as principais etapas dessa metodologia - Planejamento, Execução e Avaliação - estão sendo aplicadas nestas modalidades de ensino, bem como identificar os Ambientes Virtuais de Aprendizagem (AVA) e ferramentas de suporte geralmente mais utilizados.

Foram realizadas buscas por trabalhos científicos que apresentassem mapeamentos ou revisões sistemáticas da literatura (RSL) relacionados a Aprendizagem baseada em Problemas. Polyzois et al. (2010) conduziram uma RSL com foco na aplicação da metodologia ABP para educação de cursos da área de saúde. Merritt et al. (2017) conduziram uma RSL sobre a efetividade da ABP no Ensino de Ciências e Matemática do ensino fundamental. Jamal et al. (2019) realizou uma RSL sobre o uso de animações como estratégias de problemas da metodologia ABP. Barros Costa et al. (2018) conduziram um mapeamento sistemático da literatura sobre o ensino de programação a partir de uma abordagem baseada na resolução de problemas e jogos. De Farias et al. (2018) apresentaram uma revisão sistemática sobre o Moodle como Ferramenta de Suporte a PBL em Rede. Os trabalhos relacionados foram bastante significativos no que tange a aplicação da metodologia ABP, contudo somente um deles considerou a aplicação de ABP no Moodle, o que diferencia-se da RSL apresentada neste artigo por considerar somente um AVA, por considerar estudos até 2016 e principalmente por não ter como foco questões relacionadas ao método $\mathrm{ABP}$ no ensino a distância ou híbrido, mas sim quanto ao uso do Moodle como suporte a ABP.

\section{Método de Pesquisa}

Adotou-se a Revisão Sistemática da Literatura (RSL) como metodologia para esta pesquisa. de acordo com Sampaio e Mancini (2017), algumas das vantagens de uma revisão sistemática é a possibilidade de ser replicável e de identificar lacunas na pesquisa atual. Para a realização desta pesquisa, uma equipe de cinco integrantes executou três fases distintas: Planejamento, Construção e Resultados, com suas respectivas etapas apresentadas na Figura 1.

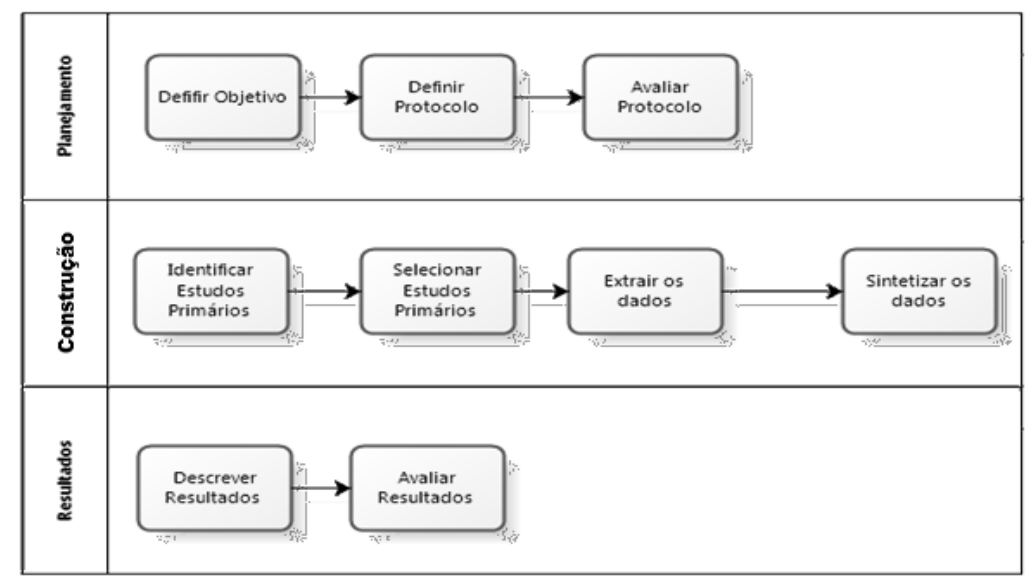

Figura 1 - Fases da Revisão Sistemática 
No Planejamento, foram definidos o objetivo e as questões de pesquisa, além da definição do protocolo. $\mathrm{Na}$ fase de Construção, procedeu-se à seleção dos estudos e à análise das questões de pesquisa, bem como à sintetização dos dados coletados. Definiu-se a string de busca nos engenhos de busca de artigos científicos mais significativos da área, selecionando-os a partir dos critérios de exclusão. Na última fase, resultados, foram tabuladas e classificadas as incidências das questões em cada artigo selecionado, utilizando a ferramenta de análise qualitativa WebQDA ${ }^{1}$.

\subsection{Objetivo e Questões de Pesquisa}

O objetivo central deste trabalho foi o de compreender como a metodologia ativa de Aprendizagem baseada em Problemas vem sendo aplicada em cursos da modalidade a distância e híbrida, bem como quais os suportes ferramentais para aplicação dessa metodologia. Para tanto, para alcançar esse objetivo, foram elaboradas as questões de pesquisa: (Q1) Como as três principais fases da metodologia ativa ABP (planejamento, execução ou aplicação e avaliação) podem ser aplicadas na EaDH? (Q2) Quais os Ambientes Virtuais de Aprendizagem que suportam de alguma forma a aplicação do ABP na EaDH? (Q3) Dos trabalhos que utilizam o Moodle como plataforma para aplicação de $\mathrm{ABP}$ na $\mathrm{EaDH}$, quais são as ferramentas ou plugins que oferecem esse suporte? (Q4) Quais os principais impactos positivos da aplicação da metodologia ABP na EaDH?

\subsection{Estratégia de busca}

$\mathrm{Na}$ construção da string de busca, foram identificados os constructos que deveriam estar contidos nos trabalhos pesquisados, em seguida foram identificados os sinônimos e ou termos alternativos dessas palavras-chaves. A tabela 1 apresenta a string gerada.

Tabela 1. String de busca

("PBL" OR "Problem-based Learning") AND ("distance learning” OR "e-learning" OR "blended learning" OR "hybrid learning") AND ("learning management system" OR "online learning environment" OR "virtual learning environment" OR "collaborative learning environment" OR "collaborative learning tool")

\subsection{Coletas dos trabalhos e Fontes de Busca}

A coleta dos trabalhos ocorreu em duas etapas: a busca nas fontes de dados digitais entre os anos de 2008 a 2019 e a seleção dos trabalhos de acordo com os critérios de exclusão. O planejamento inicial era considerar o intervalo de 2010 a 2019, mas baseado em uma busca ad-hoc no Google Scholar utilizando a string de busca, estudos relevantes publicados em 2008 e 2009 foram retornados, o que justificou a extensão do alcance da busca deste RSL para 12 (doze) anos. Foi necessário traduzir a string de busca para a língua portuguesa de modo a conduzir as pesquisas na Revista Brasileira de Informática na Educação (RBIE) e no Simpósio Brasileiro de Informática na Educação (SBIE). As bibliotecas digitais utilizadas na busca automatizada, com a quantidade de artigos indexados, foram: Elsevier ScienceDirect com 391 artigos; ACM Digital Library, 87; IEEEXplore Digital Library, 35; SBIE - Simpósio Brasileiro de Informática na Educação, 39 e RBIE - Revista Brasileira de Informática na Educação com 81 artigos retornados. Um total de 633 artigos foram indexados no Mendeley. As fontes de buscas utilizadas para realização desse trabalho foram escolhidas pelo grau de relevância científica considerando a área de concentração da pesquisa e pela acessibilidade. É importante destacar que o motor de busca presente nestas

1 https://www.webqda.net/ 
IX Congresso Brasileiro de Informática na Educação (CBIE 2020)

Anais do XXXI Simpósio Brasileiro de Informática na Educação (SBIE 2020)

fontes apresenta opções de parametrização que permitem o filtro mais detalhado dos trabalhos pesquisados.

\subsection{Critérios de Exclusão}

Para a inclusão de um trabalho na pesquisa, foi determinada sua relevância em relação às questões de pesquisa considerando os critérios de exclusão, a saber: (i) trabalhos incompletos; (ii) trabalhos duplicados; (iii) trabalhos publicados em idiomas diferentes do inglês ou português; (iv) trabalhos que não fornecem indicações de como a metodologia ativa de aprendizagem ABP é aplicada no contexto da educação a distância ou híbrida; e (v) trabalhos que não são estudos primários.

Após a aplicação dos critérios de exclusão pela leitura sistematizada dos títulos, palavras chaves, resumos e eventualmente do corpo do trabalho, dos 633 trabalhos retornados na busca realizada, 77 deles foram incluídos para a etapa de extração dos dados. Para que o estudo fosse incluído, foi necessário que dois pesquisadores o aprovassem após análise minuciosa, considerando os critérios de exclusão. Caso houvesse discordância, um terceiro pesquisador atuaria como árbitro. A tabela 3 apresenta a quantidade de trabalhos incluídos por engenho de busca. Todos os trabalhos incluídos podem ser encontrados no link https://cutt.ly/goJ3aHP.

Tabela 3. Trabalhos selecionados, Incluídos e Excluídos.

\begin{tabular}{|l|l|l|l|}
\hline Fontes de busca & Selecionados & Incluídos & Excluídos \\
\hline IEEE Xplore & 35 & 17 & 18 \\
\hline ACMDigital & 87 & 6 & 81 \\
\hline ScienceDirect & 391 & 40 & 350 \\
\hline SBIE & 39 & 11 & 28 \\
\hline RBIE & 81 & 3 & 78 \\
\hline
\end{tabular}

\subsection{Extração e Sintetização dos Dados}

Após a seleção dos estudos, os artigos foram submetidos à fase de extração e sintetização dos dados, na qual foram lidos por completo e extraídos de seus conteúdos as possíveis respostas e/ou relações com as questões de pesquisa. Nesta etapa, dois pesquisadores, de forma independente, realizaram a análise e compararam os resultados através de um processo de junção (merge). Conflitos foram solucionados por consenso através de reuniões para se estabelecer concordância. A ferramenta utilizada para a síntese e análise qualitativa das questões de pesquisa foi a WebQDA.

\section{Análise das questões de pesquisa}

Esta seção mostra o mapeamento das evidências encontradas nos estudos primários como respostas aos questionamentos levantados nas quatro questões propostas.

\section{Q1 - Como as três principais fases da metodologia ativa ABP (planejamento, execução} ou aplicação e avaliação) podem ser aplicadas na EaDH?

Dos trabalhos que responderam à Questão 1, seis abordaram o planejamento; nove a fase de execução; dois a fase de avaliação; e doze consideraram todas as fases. Entre os doze trabalhos que tratam das três fases da metodologia ABP, destacam-se Sein-echaluce et al (2015), que descreve a análise de uma experiência sobre a adaptabilidade à EaD da metodologia ABP. A experiência foi realizada com dois grupos distintos de alunos, uma com o primeiro ano de Engenharia Mecânica e outra com o quarto ano de Engenharia 
Mecatrônica. Para essa experiência, as três fases da ABP foram aplicadas, utilizando-se o ambiente de aprendizagem Moodle. Na proposta, o Planejamento consistiu na definição do problema, das equipes e das técnicas para suporte a adaptabilidade requerida. Na fase de execução os alunos realizam as tarefas propostas de acordo com o planejamento. Somente após a execução e avaliação de uma tarefa do problema definido é que se pode passar para a próxima. Para facilitar a comunicação e a colaboração, utilizaram ferramentas como wiki, fórum e chats. É importante destacar que a fase de avaliação foi cíclica através dos feedbacks dos alunos sobre a qualidade do treinamento.

Natassha e Azizah (2015) apresentam o plugin DBdesign, que contempla 14 princípios definidos por Connolly e Begg (apud Natassha e Azizah 2015), e que dá suporte as fases de planejamento, execução e validação da ABP. O DBdesign permite que no planejamento as seguintes atividades sejam desenvolvidas: criação da tarefas e definição do problema baseado em seis tipos de habilidades cognitivas, fornecimento de uma lista de competências na descrição das tarefas e o fornecimento de materiais de aprendizagem (slides, texto, vídeo, som, etc); Na execução, permite o envio das tarefas para os alunos do mesmo grupo, realização de anotações nas tarefas o compartilhamento das atividades, interações entre os grupos e entre grupos e professores e discussão em fórum e em e salas de chats. No módulo de avaliação existe a possibilidade de premiação em forma de emblemas ou pontuação extra para os alunos, de feedback ao final de cada tarefa e de avaliação do resultado dos trabalhos dos alunos.

Santos et al (2011) apresentam e validam um sistema para planejamento e compartilhamento do problema que fará parte do processo de aprendizado. O sistema disponibiliza módulos distintos: Para o planejamento existe o módulo de descrição dos problemas, orientações sobre o problema, metas de aprendizagem, orientações para o tutores dentre outros recursos. Para a execução encontra-se o compartilhamento dos problemas, edição de texto de forma colaborativa e inserção de imagens. Para a fase de avaliação o sistema permite definir os critérios que serão utilizados na avaliação.

Rodrigues e Santos (2013) apresentam uma proposta de gerenciamento das fases de planejamento, execução, acompanhamento e avaliação para a aplicação da metodologia ABP no AVA Amadeus. Conforme o escopo do trabalho apresentado pelos autores, duas fases foram abordadas: a fase de planejamento e a fase de acompanhamento. $\mathrm{O}$ Planejamento envolverá todas as ações que deverão ser executadas no processo. Para tanto foi proposto uma tela para cadastro dos módulos de aprendizagem, que conterá os conteúdos abordados, a definição do problema, o estabelecimento dos objetivos educacionais e as estratégias de avaliação. A fase de acompanhamento e visa verificar se os objetivos educacionais foram atingidos. Para esta fase foram definidas as seguintes atividades: definição da avaliação, mapeamento dos objetivos educacionais, verificação dos resultados e divulgação dos feedbacks

\section{Q2 - Quais os Ambientes Virtuais de Aprendizagem que suportam de alguma forma a aplicação do ABP na EaDH?}

Ao analisar a questão 2 foi possível identificar alguns AVAs desenvolvidos ou utilizados no suporte à utilização da metodologia ABP na EaDH. Oliveira e Santos (2016) desenvolveram um AVA de propósito específico para suporte ao planejamento, gerenciamento, monitoramento e documentação do fluxo de trabalho utilizando metodologia ABP. De acordo com os autores, o PBLMaestro, além de mecanismos de gamificação e de Learning Analytics, também oferece soluções para algumas questões 
IX Congresso Brasileiro de Informática na Educação (CBIE 2020)

Anais do XXXI Simpósio Brasileiro de Informática na Educação (SBIE 2020)

presentes na aplicação da metodologia. O PBLMaestro foi validado por meio de um estudo de caso com alunos do curso de Ciência da Computação, sendo possível identificar um alto grau de satisfação no que diz respeito a usabilidade e consistência da ferramenta.

Luis et al. (2015) apresentam uma experiência com um grupo de 55 alunos de Engenharia da universidade de La laguna. Foram utilizados o AVA Moodle como centro de organização e comunicação das atividades, os apps do Google para as atividades colaborativas, o Prezzi para apresentações, a ferramenta Webvideo para criação e edição dos vídeos, as ferramentas dipity para utilização de uma barra cronológica interativa e do piktochart para criação de infográficos. Os autores consideraram o processo de aprendizagem com o uso de tecnologias móveis e Internet adequado para a aplicação da metodologia ABP.

Melo-Solarte e Baranauskas (2008) analisaram a aplicação de cinco AVAs: Atutor, Fle3, Moodle, Sakai e Teleduc para implementação do modelo ACDP (Aprendizagem Colaborativa Baseada em Problemas), que incorpora a articulação das metodologias ABP e CSCL (Computer Supported collaborative Learning). Constatou-se que somente o Fle3 não tinha suporte para $80 \%$ das atividades propostas pelo modelo ACDP e que nenhum AVA apresentou recurso para apoiar o processo de análise e clarificação de problemas e o design do processo de comunicação, não favorecendo com isso, as discussões e reflexões nos grupos. Diante desse contexto, os autores optaram por utilizar o SAKAI para a implementação do modelo, pois o mesmo apresentou dentre outros recursos, uma ferramenta que permitiu a utilização de links externos de maneira mais acessível que os demais sistemas.

Sancho-Thomas et al. (2009) analisam três estudos de casos realizados em cursos universitários utilizando a ferramenta NUCLEO incorporado ao LMS Moodle. Os autores pesquisaram a aplicação e identificação das habilidades de trabalho em equipe, norteada por uma prática pedagógica de ABP. O NUCLEO é uma ferramenta para um contexto de aprendizado misto com dois objetivos básicos: promover a aquisição de habilidades de trabalho e habilidades sociais e, encorajar o aluno a adotar um papel mais ativo. De acordo com os autores, foi possível perceber um maior engajamento e motivação dos alunos com a utilização da ferramenta para a execução das atividades definidas pela metodologia ABP.

Dos 77 artigos analisados, 18 (dezoito) utilizaram o Moodle como Ambiente Virtual de Aprendizagem, mesmo que através de uma ferramenta específica incorporada ao mesmo, tais como DBDesign (Nattassha \& Azizah, 2015), ePBL (Ali et al. ,2015) e NUCLEO (Sancho, P. et al. 2011). Melo-Solarte \& Baranauskas (2009) analisaram os AVAs Atutor, Sakai, Teleduc e Fle3 quanto ao suporte à metodologia ACBP (Aprendizagem Colaborativa Baseada em Problemas). Outros AVAs citados nos estudos incluídos foram: ILIAS (Eichler et al, 2013), Blackboard (Ioannou et al., 2015) e Amadeus (Rodrigues \& Santos, 2013). Alguns estudos propõem AVAs específicos baseados na metodologia ABP, tais quais OPBCL (Nadiyah \& Faaizah, 2015), WoBaLearn (Zhang et al., 2016), Toth (Acosta, 2016), PBL-Coach (Bessa et al., 2017) e PBLMaestro (De Oliveira \& Santos, 2016).

Uma quantidade considerável de trabalhos, 58 (cinquenta e oito), não utilizaram AVAs como ambientes para aplicação da ABP na Educação a Distância ou Híbrida. Nesses trabalhos foram descritas estratégias de Aprendizagem baseada em Problemas na EaDH utilizando ferramentas síncronas e assíncronas de trocas de mensagens, redes sociais, 
ferramentas de edição colaborativa de documentos, ferramentas de programação, além de jogos educacionais.

\section{Q3 - Dos trabalhos que utilizam o Moodle como plataforma para aplicação de ABP na EaDH, quais são as ferramentas ou plugins que oferecem esse suporte?}

De acordo com a plataforma Capterra (2020), o AVA Moodle é a plataforma com mais usuários no mundo, além de ser o AVA mais utilizado nas Universidades e Institutos Federais vinculados à Universidade Aberta do Brasil (UAB). Considerando isso e também o alto percentual de trabalhos incluídos nesta RSL que utilizaram o Moodle, analisamos nesta questão de pesquisa as ferramentas nativas e plugins do Moodle que dão suporte a aplicação da metodologia $\mathrm{ABP}$ na EaDH. A figura 2 apresenta as ferramentas mais utilizadas.

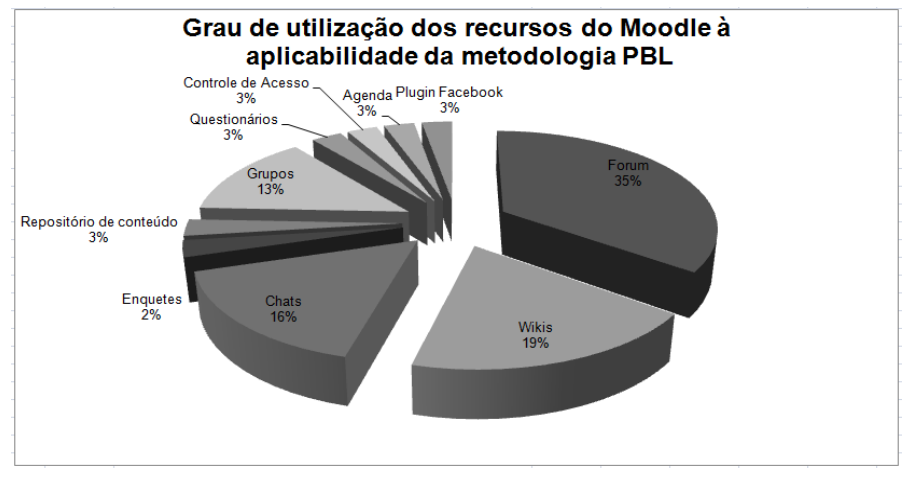

Figura 2. Incidência de ferramentas e plugins do Moodle

Tirado e Santos (2014) apresentam um estudo de caso realizado com 36 alunos do curso de Psicologia, que teve como objetivo verificar a intensidade e a qualidade de ensino baseado na metodologia ABP a partir do uso de ferramentas fornecidas pelo Moodle. Para o estudo foi proposta à utilização das ferramentas de comunicação Chat, Fórum de Discussão e Wiki. A validação deste estudo foi realizada através de questionários de avaliação e, segundo os autores, os resultados demonstraram que as equipes que trabalharam com mais colaboração obtiveram melhor índice de aproveitamento.

Os autores Foo e Hussain (2010) propõem uma investigação acerca do fortalecimento da aprendizagem autodirigida por meio de atividades colaborativas apoiadas por diversos recursos tecnológicos, tais como: fóruns de discussão on-line do Moodle, Google Docs e Wikis. Conforme apresentado pelos autores, a metodologia ABP incorpora a aprendizagem autodirigida ao requerer do aluno uma capacidade autônoma de gerenciar seu próprio processo de aprendizagem. Foi conduzido um estudo de caso com 14 alunos do curso de Mestrado em Tecnologia da Educação, que utilizaram durante um semestre as diversas ferramentas. Neste estudo, o Moodle foi utilizado para compartilhamento dos artefactos gerados e para as comunicações formais através dos fóruns de discussões. Este estudo apontou que as estratégias e ferramentas utilizadas contribuíram positivamente para na promoção de habilidades de trabalho em equipe, colaboração, organização, responsabilidade, auto regulação da aprendizagem e pensamento crítico.

Ioannou, Brown e Artino (2015) publicaram um estudo sobre o comportamento dos alunos no uso de Wiki e de fóruns de discussão para realizar atividades colaborativas baseados em problemas. Foi projetado um bloco para permitir o uso simultâneo de Wiki e fórum de discussão em uma única tela. Segundo os autores, após análise realizada em dois níveis: codificação e contagem das contribuições dos alunos com relação às evidências por 
IX Congresso Brasileiro de Informática na Educação (CBIE 2020)

Anais do XXXI Simpósio Brasileiro de Informática na Educação (SBIE 2020)

diferenças no uso das tecnologias, o estudo revelou a ferramenta Wiki como mais apropriada no contexto colaborativo de resolução de problema.

\section{Q4 - Quais os principais impactos positivos da aplicação da metodologia ABP na EaDH?}

A análise da questão quatro permitiu aferir que muitos estudos atestam impactos positivos do uso da metodologia $\mathrm{ABP}$ na $\mathrm{EaDH}$, dentre esses impactos estão o aumento no grau de motivação dos alunos, diminuição da evasão, desenvolvimento das competências de liderança, comunicação, pensamento crítico e inovador, resolução de problemas, autorregulação de aprendizagem e colaboração.

Garcia-Robles, et al (2009) apresentam um estudo de caso da aplicação da metodologia ABP com uma abordagem orientada ao design, baseada no protocolo Learning Design (IMS-LD). O estudo foi conduzido em um curso de Engenharia e utilizou o AVA Moodle como suporte operacional. Após a aplicação de dois questionários que envolviam questões sobre o ambiente de ensino e sobre a metodologia ABP, foi possível identificar como pontos de impactos positivos a satisfação dos alunos envolvidos no processo, a melhor capacidade de divisão de responsabilidades pelos alunos e uma melhor estruturação das atividades por parte dos professores utilizando o AVA Moodle.

Sancho et al (2011) abordam um estudo da aplicação da metodologia ABP com 300 alunos de uma Universidade espanhola. Nesta experiência utilizou-se o plugin NUCLEO juntamente com o AVA Moodle. O NUCLEO se baseia em uma estratégia subjacente a ABP, que combina jogos dinâmicos no processo de ABP. A experiência objetiva uma mudança na atitude dos alunos tornando-os mais ativos por meio de jogos de metáfora e de dinâmicas de role-playing, melhorando as habilidades sociais através de formação de equipes semi-autônomas. Os resultados foram obtidos automaticamente através da interação dos alunos, onde as variáveis utilizadas analisaram o compromisso, comunicação, inovação e a habilidade de liderança de cada aluno, sendo considerados satisfatórios e replicáveis.

Vivian et al (2016) conduziram uma pesquisa com 23 alunos do curso de Ciência da Computação da Universidade de Adelaide, objetivando a verificação da eficácia da ferramenta Wiki utilizando a metodologia ABP, testando a estrutura de trabalho em equipe e os sentimentos dos alunos durante realização das tarefas. No estudo de caso foi utilizada a ferramenta colaborativa Piazza para verificar as contribuições quanto a coesão do trabalho em equipe e análise de frequências de comportamentos cognitivos e metacognitivos. Os autores verificaram que a ABP proporcionou aos alunos a capacidade de praticar o trabalho em equipe online; aplicar os conhecimentos da disciplina; praticar habilidades de negociação e resolver problemas em grupos. O componente de discussão do Wiki forneceu um espaço valioso no qual os alunos foram capazes de negociar e construir consenso antes ou durante o desenvolvimento das soluções.

Bessa et al. (2017) descrevem um estudo de caso do uso da ABP em uma escola secundária da Índia, utilizando o AVA PBL-Coach. Segundo os autores, os impactos da aplicação retrataram um alto nível de aprendizagem dos alunos, demonstrado através de uma avaliação 360 graus. No nível experimental, durante os anos acadêmicos de 2007-2008 e 2008-2009, em três diferentes contextos do ensino superior, as taxas de abandono diminuíram em comparação aos anos anteriores. Para os autores, quatro ações justificam esses impactos: proposição pelos alunos das próprias soluções para os problemas; trabalho em grupo; criação de um protótipo para resolução do problema; e avaliação formativa. 
IX Congresso Brasileiro de Informática na Educação (CBIE 2020)

Anais do XXXI Simpósio Brasileiro de Informática na Educação (SBIE 2020)

Sein-Echaluce et al (2015) apresentam uma experiência de ABP com a utilização de diversas ferramentas do AVA Moodle, tais como: questionário, fórum, grupos de trabalhos, controle de acesso e de atividades. Neste trabalho os autores identificaram como principal impacto da aplicação da ABP o aprimoramento das competências individuais e em grupo.

\section{Conclusões}

Esta Revisão Sistemática da Literatura confirma que a ABP é utilizada além dos ambientes de aprendizagem presenciais. A RSL analisou 77 estudos que aplicaram ABP necessariamente na modalidade a distância ou híbrida, dos quais aproximadamente $75 \%$ descreveram uma pesquisa ou experiência utilizando APB sem vinculá-la a um Ambiente Virtual de Aprendizagem ou mesmo a uma ferramenta de propósito específico de ABP. Nesses trabalhos os autores aplicaram ABP utilizando ferramentas síncronas e assíncronas de trocas de mensagens, redes sociais, ferramentas de edição colaborativa de documentos na nuvem, ferramentas de programação, além de jogos educacionais. Uma hipótese para o grande número de trabalhos que utilizaram ferramentas externas, não integradas ao AVA, para condução de $\mathrm{ABP}$ na $\mathrm{EaDH}$, é a baixa oferta de ferramentas que promovam o trabalho em grupo e colaborativo nos AVAs analisados, característica indispensável para aplicação da metodologia da APB.

Há na literatura poucas iniciativas de ferramentas externas ou integradas aos AVAs que sejam apropriadas e específicas ao planejamento, execução ou avaliação de ABP em cursos a distância ou mesmo híbridos. Essa pesquisa buscou ter acesso aos AVAs de propósito específico à Aprendizagem baseada em Problemas WoBaLearn, Toth, PBLCoach e PBLMaestro, bem como aos plugins do AVA Moodle DBDesign, ePBL e NUCLEO, todos citados nesta RSL, nenhum estava disponível para download público, o que demonstra que as pesquisas científicas que conceberam algum artefacto tecnológico para o suporte de PBL na EaDH continuam indisponíveis para os atores envolvidos no processo de ensino aprendizagem.

\section{Referências}

Acosta, O. C. (2016). Recomendação de conteúdo em um ambiente colaborativo de aprendizagem baseada em projetos.

Ali, Z. F., Al-Dous, K., \& Samaka, M. (2015, March). Problem-based learning environments in Moodle: Implementation approches. In 2015 IEEE Global Engineering Education Conference (EDUCON) (pp. 868-873). IEEE.

Bessa, B. R., dos Santos, S. C., \& da Fonseca, L. (2017, October). Using a virtual learning environment for problem-based learning adoption: A case study at a high school in India. In 2017 IEEE Frontiers in Education Conference (FIE) (pp. 1-7). IEEE.

Bilgin, I., Karakuyu, Y., \& Ay, Y. (2015). The effects of project-based learning on undergraduate students' achievement and self-efficacy beliefs towards science teaching. Eurasia Journal of Mathematics, Science and Technology Education, 11(3), 469-477.

Capterra. The TOP 20 most popular LMS Software. Disponível em: < https://www.capterra.com/infographics/most-popular/learning-management-systemsoftware//>. Acesso em: 01 julho 2020.

de Barros Costa, E., \& Rocha, H. J. B. (2018). Programação numa Abordagem de Aprendizagem baseada em Resolução de Problemas e Jogos: Um Mapeamento Sistemático. SBC-Proceedings of SBGames. 
de Farias, G. F., Brito, N. M. D. M., Farias, F. J. S., \& de Souza, M. V. Moodle como Ferramenta de Suporte a PBL em Rede: Uma Revisão Sistemática. Educação Fora da Caixa: Tendências Internacionais e Perspectivas sobre a Inovação na Educação, 2018.

de Oliveira, F. S., \& Santos, S. (2016, October). PBLMaestro: A virtual learning environment for the implementation of problem-based learning approach in Computer education. In 2016 IEEE Frontiers in Education Conference (FIE) (pp. 1-9). IEEE.

de Souza Rabelo, D. S., Junior, H. L., Burlamaqui, A., Rabelo, H., Valentim, R., Rabelo, D. A., \& Silas, D. (2018). Desenvolvimento de Sistemas Computacionais Utilizando Aprendizagem Baseada em Problemas. Simpósio Brasileiro de Informática na Educação-SBIE, Vol. 29, p. 188.

Dorneles, S., Costa, C., Rigo, S., \& Barbosa, J. (2019, November). Um Modelo para Criação de Jogos Sérios e Ubíquos orientados à Aprendizagem baseada em Problemas. Simpósio Brasileiro de Informática na Educação, Vol. 30, No. 1, p. 743.

Eichler, A., Hoffmann, C., Kautz, C., \& Werner, H. (2013). Design of tutorial activities and homework assignments for a large-enrollment introductory course in control systems. IFAC Proceedings Volumes, 46(17), 43-48.

Sze-Yeng, F., \& Hussain, R. M. R. (2010). Self-directed learning in a socioconstructivist learning environment. Procedia-Social and Behavioral Sciences, 9, 1913-1917.

Garcia-Robles, R., Diaz-del-Rio, F., Vicente-Diaz, S., \& Linares-Barranco, A. (2009). An eLearning standard approach for supporting $\mathrm{PBL}$ in computer engineering. IEEE Transactions on Education, 52(3), 328-339.

Ioannou, A., Brown, S. W., \& Artino, A. R. (2015). Wikis and forums for collaborative problem-based activity: A systematic comparison of learners' interactions. The Internet and Higher Education, 24, 35-45.

Jamal, S. N. B., Ibrahim, N. H. B., \& Surif, J. B. (2019). Concept cartoon in problem-based learning: A systematic literature review analysis. JOTSE: Journal of Technology and Science Education, 9(1), 51-58.

Luis, C. E. M., Gutiérrez, J. M., \& Marrero, A. M. G. (2014). Using mobile devices and internet technologies in problem-based learning: Design of a suitable active and collaborative learning environment in engineering education. IEEE Frontiers in Education Conference (FIE) Proceedings (pp. 1-6). IEEE.

Melo-Solarte, D. S., \& Baranauskas, M. C. C. (2008, November). Uma Abordagem para EaD Baseada em Resolução de Problemas. Simpósio Brasileiro de Informática na Educação-SBIE, Vol. 1, pp. 716-725.

Merritt, J., Lee, M. Y., Rillero, P., \& Kinach, B. M. (2017). Problem-based learning in K-8 mathematics and science education: A literature review. Interdisciplinary Journal of Problem-Based Learning, 11(2).

Nadiyah, R. S., \& Faaizah, S. (2015). The development of online project based collaborative learning using ADDIE model. Procedia-Social and Behavioral Sciences, 195, 1803-1812.

Nattassha, R., \& Azizah, F. N. (2015, November). Database analysis and design learning tool based on problem/project-based learning. In 2015 International Conference on Data and Software Engineering (ICoDSE) (pp. 65-68). IEEE. 
Nova, C., \& Alves, L. (2003). Estação online: a ciberescrita, as imagens e a EAD. SILVA, Marco (Org.). Educação Online, 107-136.

Polyzois, I., Claffey, N., \& Mattheos, N. (2010). Problem-based learning in academic health education. A systematic literature review. European Journal of Dental Education, 14(1), 55-64.

Rodrigues, A. N., \& dos Santos, S. C. (2013). Aplicando a taxonomia de Bloom revisada para gerenciar processos de ensino em sistemas de aprendizagem baseada em problemas. Revista Brasileira de Informática na Educação, 21(01), 01.

Sampaio, R. F., \& Mancini, M. C. (2007). Estudos de revisão sistemática: um guia para síntese criteriosa da evidência científica. Brazilian Journal of Physical Therapy, 11, 8389

Sancho, P., Torrente, J., Marchiori, E. J., \& Fernández-Manjón, B. (2011, April). Enhancing moodle to support problem-based learning. The Nucleo experience. In 2011 IEEE Global Engineering Education Conference (EDUCON) (pp. 1177-1182). IEEE.

Sancho-Thomas, P., Fuentes-Fernández, R., \& Fernández-Manjón, B. (2009). Learning teamwork skills in university programming courses. Computers \& Education, 53(2), 517-531.

Santos, J. A. M., Pimentel, J. M., Bittencourt, J. C. N., \& Lago, R. S. N. (2010). PBL Manager: Uma ferramenta de compartilhamento de problemas para auxílio à metodologia de ensino PBL. Simpósio Brasileiro de Informática na Educação-SBIE, Vol. 1, No.

Sein-Echaluce, M. L., Aguado, P. M., Esteban-Escaño, J., Esteban-Sánchez, A., Florentín, P., Gracia-Gómez, M. C., \& Velamazán, M. Á. (2015, October). Design of adaptive experiences in higher education through a learning management system. 3rd International Conference on Technological Ecosystems for Enhancing Multiculturality, pp. 165-171.

Tirado, F., \& Santos, G. (2014, August). Examining the relationship between epistemic activity and academic achievement using of scripts and co-evaluation strategies in a VLE. 9th International Conference on Computer Science \& Education (pp. 758-762). IEEE.

Valente, J. A. (2014). A comunicação e a educação baseada no uso das tecnologias digitais de informação e comunicação. UNIFESO-Humanas e Sociais, 1(01), 141-166.

Vivian, R., Falkner, K., Falkner, N., \& Tarmazdi, H. (2016). A method to analyze computer science students' teamwork in online collaborative learning environments. ACM Transactions on Computing Education (TOCE), 16(2), 1-28.

Zhang, B., Yin, C., David, B., Xiong, Z., \& Niu, W. (2016). Facilitating professionals' work-based learning with context-aware mobile system. Science of Computer Programming, 129, 3-19. 